\title{
泡沫温拌沥青的玻璃态转变温度及低温性能
}

\author{
刘圣洁 ${ }^{1,2}$, 谢政专 ${ }^{1}$, 彭爱红 ${ }^{3}$ \\ 1) 广西道路结构与材料重点实验室, 广西南宁 530007 ；2）河海大学土木与交通学院， \\ 江苏南京 $210098 ； 3$ ） 江西省交通工程集团建设有限公司，江西南昌 330029
}

摘 要: 为研究泡沫温拌沥青的玻璃态转变温度及低温性能, 采用不同的沥青类型和不同的发泡用水 量, 制备泡沫温拌励青. 利用动态热力学分析仪 (dynamic thermomechanical analysis, DMA) 和差示扫描量热 法 (differential scanning calorimetry, DSC), 确定不同发泡水量下泡沫温拌沥青玻璃态转变温度, 采用低温弯 曲流变梁 (bending beam rheometer, BBR) 分析不同发泡水量下泡沫温拌沥青的低温性能. 结果表明，分别 通过 DMA 和 DSC 确定的玻璃态转变温度有所区别，但对于不同的励青和发泡用水量，两者的变化趋势一 致; 随着发泡水增多, 沥青玻璃态转变温度逐渐增高; 加入发泡水会在一定程度降低沥青的低温抗裂性能, 采用质量分数为 $6 \%$ 的发泡水制备的泡沫温拌励青不能满足 $-12{ }^{\circ} \mathrm{C}$ 时的低温环境要求，也无法满足原有未 发泡沥青的低温环境适用范围。为保证泡沫温拌励青良好的路用性能，泡沫温拌沥青制备时，最佳发泡用 水量选择应重点考虑其低温抗裂能力.

关键词: 路面工程; 沥青材料; 泡沫温拌沥青; 玻璃态转变温度; 低温性能; 动态热力学分析; 差示 扫描量热

中图分类号：U414 文献标志码：A doi : 10.3724/SP. J. 1249.2021.02163

\section{Glass transition temperature and low temperature performance for foam warm mix asphalt}

\section{LIU Shengjie ${ }^{1,2}$, XIE Zhengzhuan ${ }^{1}$, and PENG Aihong ${ }^{3}$}

1) Guangxi Key Laboratory of Road Structure and Materials, Nanning 530007, Guangxi Zhuang Autonomous Region, P. R. China

2) College of Civil and Transportation Engineering, Hohai University, Nanjing 210098, Jiangsu Province, P. R. China

2) Jiangxi Communications Engineering Group Construction Co. Ltd. , Nanchang 330029, Jiangxi Province, P. R. China

\begin{abstract}
In order to study the glass transition temperature and low temperature performance of foam warm mix asphalt (FWMA), we have prepared the FWMA samples with different asphalt types and different foaming water contents $(\mathrm{FWC})$. Glass transition temperatures of the samples are determined by using dynamic mechanical analyzer (DMA) and differential scanning calorimetry (DSC) methods, and their low temperature performancesare evaluated by using bending beam rheometer ( BBR) method. Results show that for different asphalt types and FWC, the behaviors of glass transition temperatures determined by DMA and DSC are different, but their changing trends are
\end{abstract}

Received: 2020-03-26; Revised: 2020-12-06; Accepted: 2021-01-15

Foundation: National Natural Science Foundation of China ( 51908194 ); China Postdoctoral Science Foundation Project ( 2019M650101 ); Key Research and Development Program of Guangxi Zhuang Autonomous Region (20192BBG70080); Key Research and Development Program of Jiangxi Province (2020AB42005)

Corresponding author: Associate professor LIU Shengjie. E-mail: lsjwork@126.com

Citation: LIU Shengjie, XIE Zhengzhuan, PENG Aihong. Glass transition temperature and low temperature performance for foam warm mix asphalt $[\mathrm{J}]$. Journal of Shenzhen University Science and Engineering, 2021, 38(2) : 163-169. (in Chinese) 
similar. With the increase of FWC, the glass transition temperature of asphalt increases gradually. The addition of foam water damages the low temperature cracking resistance of asphalt to a certain extent. Foaming water with a mass fraction of $6 \%$ could not meet the low temperature requirement below $-12{ }^{\circ} \mathrm{C}$, and could not satisfy the application scope of the non-foaming asphalt at low temperature. In order to ensure good pavement performance of FWMA, the low temperature cracking resistance must be taken as the priority consideration for choosing the optimal FWC.

Key words : pavement engineering; asphalt materials; foam warm mix asphalt; glass transition temperature; low temperature performance; dynamic thermomechanical analysis; differential scanning calorimetry

随着 “绿色交通”和 “低碳减排” 理念逐渐 成为社会关注的焦点和未来发展的趋势, 如何促进 沥青路面施工过程向节能、环保和低能耗的方向发 展，建设以低碳减排为核心的绿色铺面技术，已成 为当前道路界面临的重大课题. 泡沫温拌沥青技术 无需添加任何化学添加剂, 仅通过注人微量水分使 沥青发泡，即可有效降低施工温度，实现沥青路面 的施工低碳化 ${ }^{[1]}$. 由于出色的降温效果和经济性 能, 泡沫温拌沥青 (foamed warm mix asphalt, FWMA) 混合料获得了突飞猛进的发展应用. 根据美 国沥青路面协会 (National Asphalt Pavement Association，NAPA）统计，2017 年在全美范围内 FWMA 占温拌沥青混合料 (warm mix asphalt, WMA) 总量的 $64.7 \%$. 因此，从国外温拌沥青技术应用及发展 来看, 泡沫温拌沥青技术是以后发展的趋势.

沥青是典型的温度敏感性材料, 其力学性能直 接依赖于外界环境. 在不同温度下沥青表现为 3 种 相态, 即高温时的黏流态、中温时的黏弹态和低温 时的玻璃态. 通常将沥青材料由黏弹态转变为玻璃 态时的临界温度称为玻璃化转变温度 ${ }^{[3]}$. 研究发 现, 沥青的物理性能和化学性能在玻璃化转变温度 发生了急剧的变化 ${ }^{[4]}$. 从分子性能来看, 沥青的玻 璃态转变温度是高分子从冻结到运动的一个转变温 度，在玻璃态转变温度以下，沥青分子链段的运动 处于冻结状态，外力作用下的变形难以快速恢复和 有效松弛，容易引起沥青材料开裂 ${ }^{[5]}$. 因此，目前 普遍认为, 沥青的玻璃态转变温度与其低温性能密 切相关, 理想的状态是沥青的玻璃态转变温度低于 其最低服役温度，从而保证其在使用过程中保持良 好的变形性能，松驰掉内部集聚的应力，避免路面 产生开裂. 研究沥青的玻璃态转变温度具有丰富的 工程意义 ${ }^{[6-7]}$.

国内外学者针对常规沥青的玻璃态转变温度开 展了广泛的研究, 也取得丰硕的成果 ${ }^{[8-10]}$. 但由于 FWMA 的生产过程短暂且拌合温度较低，发泡过程
注人的水分难以全部排除，致使泡沫温拌沥青性能 与常规沥青不同 ${ }^{[11]}$. 目前普遍认为 FWMA 路面的 水稳定性会在一定程度上降低, 且 FWMA 的低温 性能也略低于 $\mathrm{WMA}^{[12-13]}$. ALHASAN 等 ${ }^{[14]}$ 发现, 泡沫温拌沥青的低温性能早期也弱于普通沥青. 玻 璃化转变温度的确定对沥青及沥青混合料内温度应 力的预测、低温开裂的控制有十分重要的意义. 但 目前针对泡沫温拌沥青玻璃态转变温度的研究较 少. 为深人探索泡沫温拌沥青的相态转变行为, 考 察不同发泡水量对其低温性能的影响，本研究采用 动态热力学分析 (dynamic thermomechanical analysis, DMA) 仪和差示扫描量热 ( differential scanning calorimetry, DSC) 仪，对比测定泡沫温拌沥青的玻璃态 转变温度, 分析发泡用水量对玻璃态转变温度的影 响, 并采用低温弯曲流变梁 (bending beam rheometer, BBR) 试验分析不同发泡水量下泡沫温拌沥青 的低温性能.

\section{1 试验材料及方法}

\section{1 原材料}

采用 $70^{\#}$ 基质沥青和 SBS 改性沥青. 基质沥青 的针人度 $\left(25^{\circ} \mathrm{C}\right)$ 为 $69.5(0.1 \mathrm{~m})$, 软化点温度为 $48{ }^{\circ} \mathrm{C}$, 延度 $\left(15{ }^{\circ} \mathrm{C}\right)$ 大于 $150 \mathrm{~cm}$; SBS 改性沥青的 针人度 $\left(25{ }^{\circ} \mathrm{C}\right)$ 为 $50.4(0.1 \mathrm{~m})$, 软化点为 83.8 ${ }^{\circ} \mathrm{C}$, 延度 $\left(5^{\circ} \mathrm{C}\right)$ 为 $35.8 \mathrm{~cm}$. 发泡采用饮用水, $\mathrm{pH}$ 值为 7.95 , 游离余氯的含量为 $0.1 \mathrm{mg} / \mathrm{L}$, 可溶性 固体总量为 $186 \mathrm{mg} / \mathrm{L}$.

\section{2 泡沫温拌沥青的制备}

本研究采用室内小型发泡机 (WLB 10) 制备泡 沫温拌沥青. 将基质沥青和 SBS 改性沥青分别加热 至 $155{ }^{\circ} \mathrm{C}$ 和 $165{ }^{\circ} \mathrm{C}$. 当两种沥青呈流动状态时, 分 别注人质量分数为 $0 、 3 \%$ 和 $6 \%$ 的发泡水，共得到 6 种沥青样品. 为保证试验结果精确性和可靠性, 每种沥青开展 3 组平行试验, 取平均值作为试验 
结果.

\section{3 试验方法}

\subsubsection{DMA 试验}

玻璃态转变温度采用 DMA ( Q800) 动态力学分 析仪对沥青进行温度扫描试验. 试验时, 加载方式 为双悬臂弯拉模式, 温度范围为 $(-60 \sim 80){ }^{\circ} \mathrm{C}$, 升 温速率为 $10{ }^{\circ} \mathrm{C} / \mathrm{min}$, 扫描应变为 $0.01 \%$, 扫描频 率为 $10 \mathrm{~Hz}$.

DMA 试件的尺寸为 $60 \mathrm{~mm} \times 13 \mathrm{~mm} \times 3.5 \mathrm{~mm}$. 试验制备时，首先将发泡后的沥青浇筑于铝制模具 中, 待其自然降温为室温 $\left(20^{\circ} \mathrm{C}\right)$ 时, 将模具放人 $-60{ }^{\circ} \mathrm{C}$ 的冷冻箱中冷冻 $2 \mathrm{~h}$, 而后将冰冻沥青从模 具中取出, 置于 DMA 设备的夹具中, 开展 DMA 试 验，测得模量和相位角.

\subsubsection{DSC 试验}

采用 TA DSC25 设备开展差示扫描量热仪试验, 试验温度范围为 $(-60 \sim 130)^{\circ} \mathrm{C}$, 升温速率为 10 ${ }^{\circ} \mathrm{C} / \mathrm{min}$, 样品质量为 $10 \mathrm{mg}$, 氮气作为保护气, 流 量为 $100 \mathrm{~mL} / \mathrm{min}$. 根据泡沫温拌沥青在升温过程中 的吸热和放热特征, 获取其玻璃态转变温度.

\subsubsection{BBR 试验}

采用旋转薄膜烘箱 (rolling thin film ovens, RTFO) 试验和压力老化容器 ( pressure aging vessel, PAV) 试验, 对泡沫温拌沥青进行老化, 利用 TEBBR Pro 设备开展低温性能试验. BBR 试验时样品 尺寸为 $125 \mathrm{~mm} \times 12.5 \mathrm{~mm} \times 6.25 \mathrm{~mm}$, 试验温度分 别为 $-24 、-18$ 和 $-12{ }^{\circ} \mathrm{C}$, 记录泡沫温拌沥青的劲 度模量 $(S)$ 和蠕变速率 $(m)$.

\section{2 试验结果及分析}

\section{1 DMA 试验结果分析}

DMA 试验对沥青样品施加外部作用力后, 沥 青材料会出现变形，外部能量一部分被储存，另一 部分由于分子链段内耗而损耗, 分别对应储存模量 和损耗模量 $^{[15]}$. DMA 分析中, 当温度低于玻璃化 温度时, 分子链段很难运动, 损耗产生的能量很 少. 当温度高于玻璃化温度时, 分子链段很容易运 动, 损耗产生的能量也少 ${ }^{[16]}$. 只有玻璃化转变区 时, 分子链段能运动, 但又跟不上应力的变化, 产 生的滞后最多，损耗掉的能量最大.

温度升高时，沥青相态由玻璃态向黏弹态和黏 流态转变, 模量和相位角也呈一定变化规律. 从储 存模量、损耗模量和相位角正切值的变化规律上,
可得到相应的玻璃化转变温度 ${ }^{[17]}$ 。目前沥青材料 玻璃态转变温度可采用储能模量曲线的转折点、损 耗模量峰值点和相位角 $\tan \theta$ 的峰值点判定 ${ }^{[18]}$.

图 1 为泡沫温拌沥青的 DMA 试验结果. 从图 1 可知，不同发泡水量下的 DMA 结果有所不同，表 明发泡水的引人影响了沥青的热行为. 对于不同发 泡水量下的改性沥青，损耗模量存在明显的峰值 点，未发泡的基质沥青也存在明显的损耗模量峰值 点. 尽管发泡水质量分数为 $3 \%$ 和 $6 \%$ 时，基质沥 青的损耗模量没有明确的峰值点，但有较为明显的 转折点. 由于相位角 $\tan \theta$ 没有明显的峰值点, 本 研究将损耗模量的峰值点和转折点温度作为泡沫温 拌沥青的玻璃态转变温度, 结果如表 1 .

玻璃化转变温度越低，低温性能越好，最理想 的状况是励青的玻璃态转变温度小于该地区的最低 服务温度，保证在最低服务温度下，沥青分子链不 被 “冻结”，仍能自由活动，从而松弛由于温度降 低引起的沥青温度应力, 减小沥青低温裂缝的产 生. 从表 1 发现，随着发泡用水量的增加，基质沥 青和改性沥青的玻璃态转变温度逐渐升高. 表明随 着发泡水的加人，沥青的低温性能有所降低. 原因 是沥青发泡后的水分在后期难以全部散失，部分水 分残存于沥青中，并与沥青发生某种结合，使得沥 青分子链 “冻结” 的温度提高, 即在较高的温度 下，沥青分子链才能 “解冻”，从宏观上，沥青的 玻璃态转变温度升高.

\subsection{DSC 试验结果分析}

DMA 通过沥青的动态力学力学性能获取玻璃 态转变温度, 而 DSC 则是通过沥青的热力学行为直 接获取温度转变点. 图 2 为泡沫温拌沥青的 DSC 结 果. 从图 2 可知，两种沥青发泡后，DSC 曲线发生 改变，表明发泡过程影响了沥青的热物特性. 基质 沥青和改性沥青发泡后在 $0{ }^{\circ} \mathrm{C}$ 有明显的放热峰. 由 于 $0{ }^{\circ} \mathrm{C}$ 为水分从固体到液体的转变温度，因此，相 比于未发泡的沥青而言，该处存在的曲线峰表明泡 沫温拌沥青中含有残存的水分. 而泡沫温拌沥青 DSC 曲线的改变也推测是残存发泡水引起.

在 DSC 曲线上，在通过玻璃化转变温度时， 基线向吸热方向移动，吸热曲线会产生一个台阶， 通常将台阶中的点所对应的温度作为玻璃化转变温 度. 由 DSC 方法得到的玻璃态转变温度如表 2 .

从表 2 发现, 通过 DSC 方法得到的玻璃态转变 温度均为负值，符合沥青实际服役的低温温度. 由 于时间尺度和方法不同, DSC 和 DMA 得到的玻璃态 


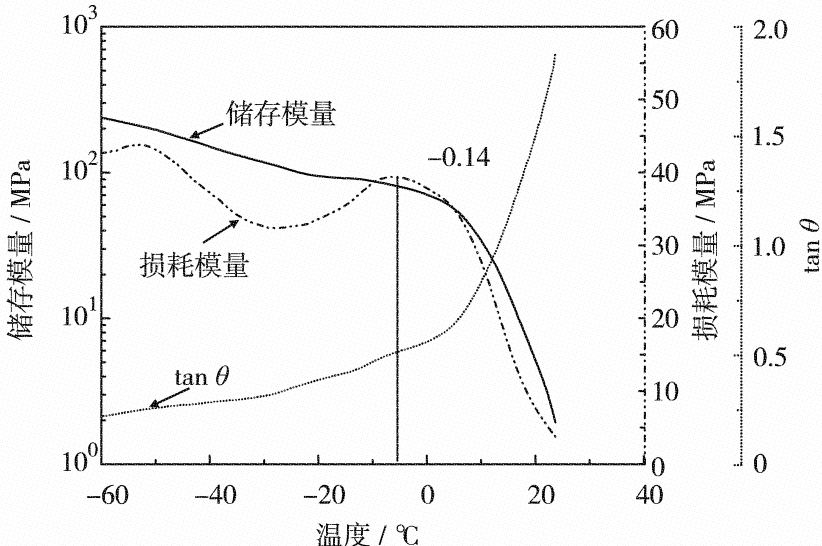

(a) 基质励青

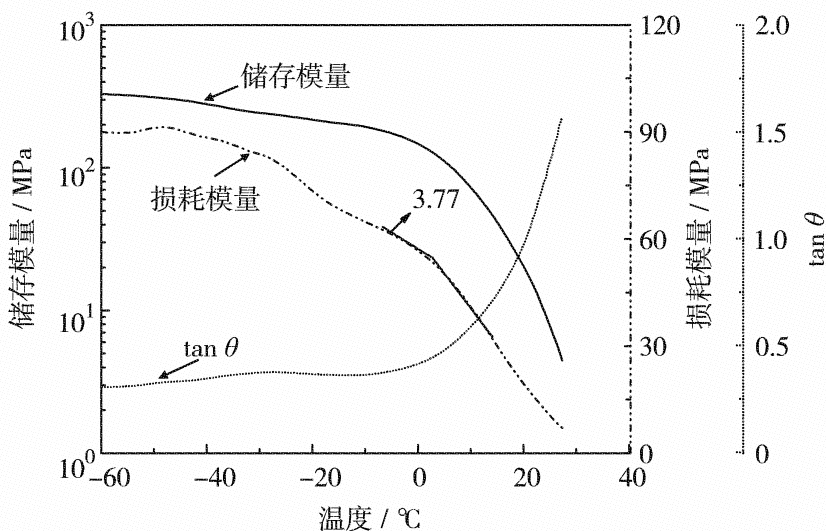

（c）泡沫温拌基质沥青 $+6 \%$ (质量分数) 发泡水

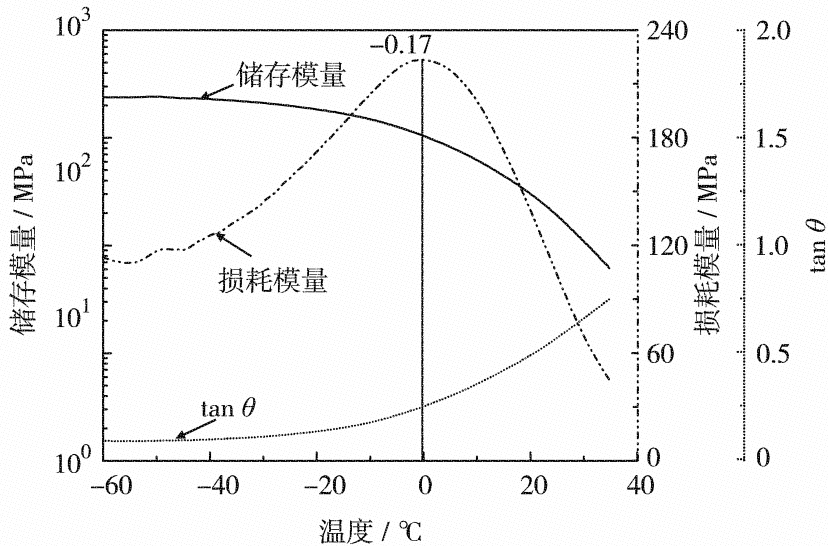

（e）泡沫温拌改性沥青 $+3 \%$ (质量分数) 发泡水

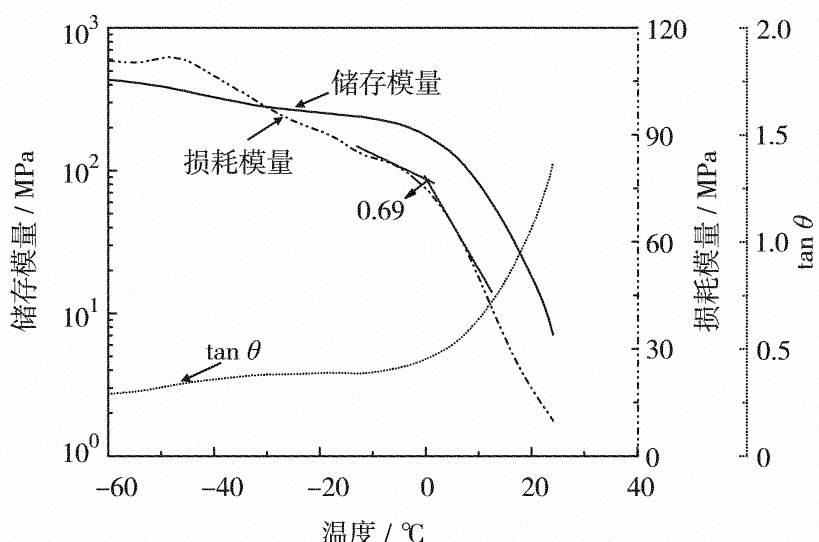

（b ) 泡沫温拌基质沥青 $+3 \%$ (质量分数) 发泡水

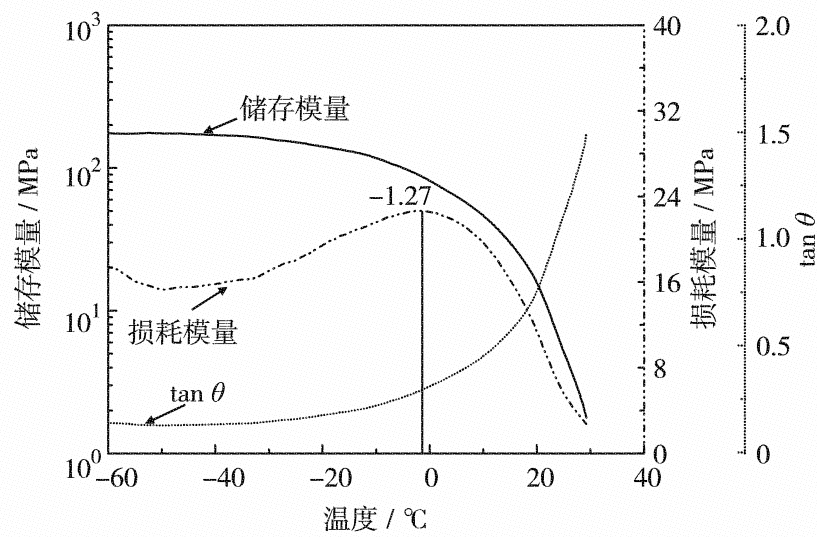

(d) 改性汸青

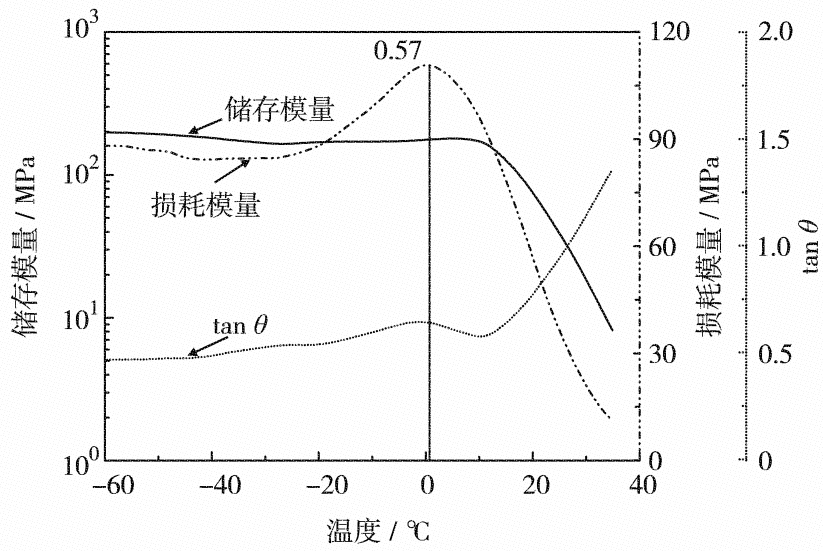

（f）泡沫温拌改性沥青 $+6 \%$ (质量分数) 发泡水

图 1 泡沫温拌沥青的 DMA 试验结果

Fig. 1 DMA test results for foam warm mix asphalt

表 1 泡沫温拌沥青玻璃态转变温度 (DMA 法)

Table 1 Glass transition temperature of foam warm mix asphalt (DMA) ${ }^{\circ} \mathrm{C}$

\begin{tabular}{lccc}
\hline 沥青类型 & 未加发泡水 & $w($ 水 $)=3 \%$ & $w($ 水 $)=6 \%$ \\
\hline 基质沥青 & -0.14 & 0.69 & 3.77 \\
改性沥青 & -1.27 & -0.17 & 0.57 \\
\hline
\end{tabular}

转变温度差别较大. 原因在于 DMA 方法中, 玻璃 态转变温度主要通过弹性模量的突变来确定, 而在 DSC 方法中, 玻璃态转变温度主要通过比热容突变 来确定. 不同的测定条件下，沥青材料分子链段的 运动性不同，因此各方法测定得到的玻璃态转变温 度具有一定差异 ${ }^{[19]}$. 因此, 在标出某种材料的玻 璃化转变温度时，必须注明测定的方法和条件，不 


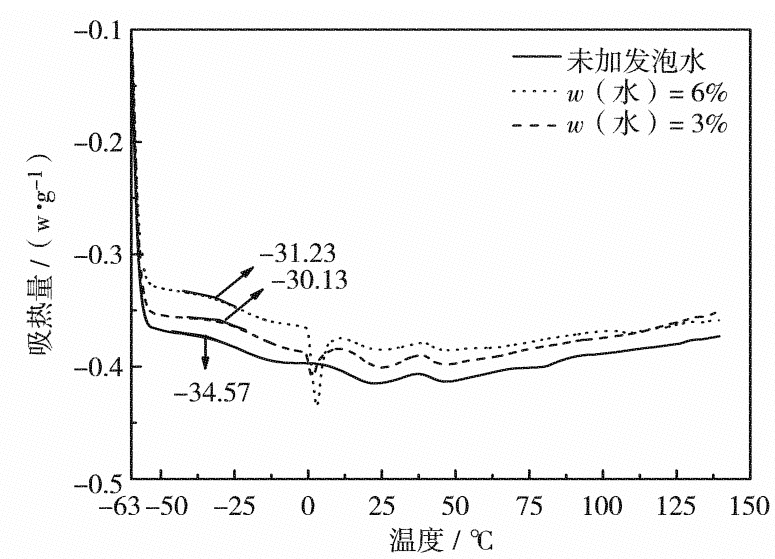

（a 泡沫温拌基质沥青

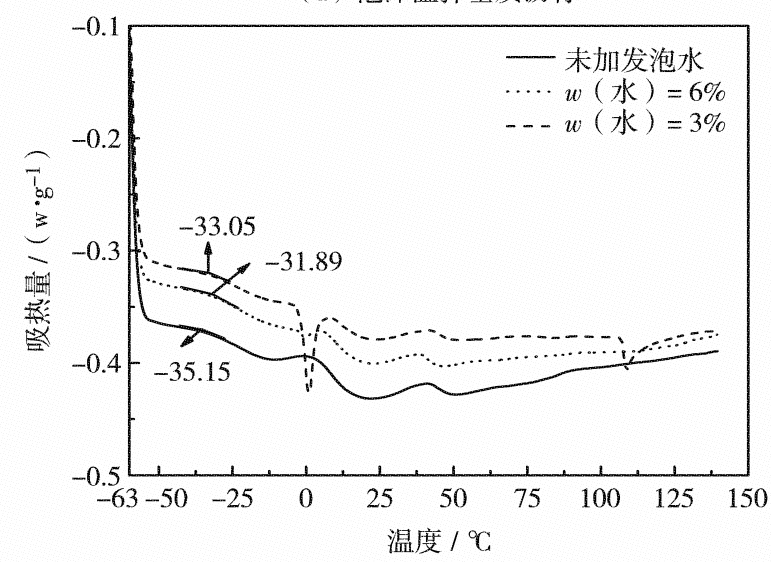

(b) 泡沫温拌改性沥青

图 2 泡沫温拌沥青的 DSC 试验结果

Fig. 2 DSC test results for foam warm mix asphalt

同方法条件之间的结果无可比性. 但从玻璃态转变 温度随发泡水量的变化趋势来看, DSC 的结果其与 DMA 的结果一致. 即随着发泡水的增多, 沥青的 玻璃态转变温度逐渐增高, 表明随着发泡量增加, 对沥青的低温性能不利.

\section{表 2 泡沫温拌沥青玻璃态转变温度 (DSC 方法)}

Table 2 Glass transition temperature of foam warm mix asphalt (DSC) ${ }^{\circ} \mathrm{C}$

\begin{tabular}{lccc}
\hline 沥青类型 & 未加发泡水 & $w$ (水) $=3 \%$ & $w$ (水) $=6 \%$ \\
\hline 基质沥青 & -34.57 & -31.23 & -30.13 \\
改性沥青 & -35.15 & -33.05 & -31.89 \\
\hline
\end{tabular}

\section{3 泡沫温拌沥青 BBR 结果分析}

根据 BBR 试验得到的 $S$ 和 $m$ 用于评价沥青的低 温抗裂性能. 通常而言, $S$ 越小，沥青的低温柔性 越大; $m$ 越大, 沥青的应力松弛能力越强, 低温抗 裂性越好. 不同发泡用水量下泡沫温拌沥青的劲度 模量和蠕变速率如图 3. 由图 3 可见, 对于基质沥 青和改性沥青而言, 劲度模量和蠕变速率随温度变
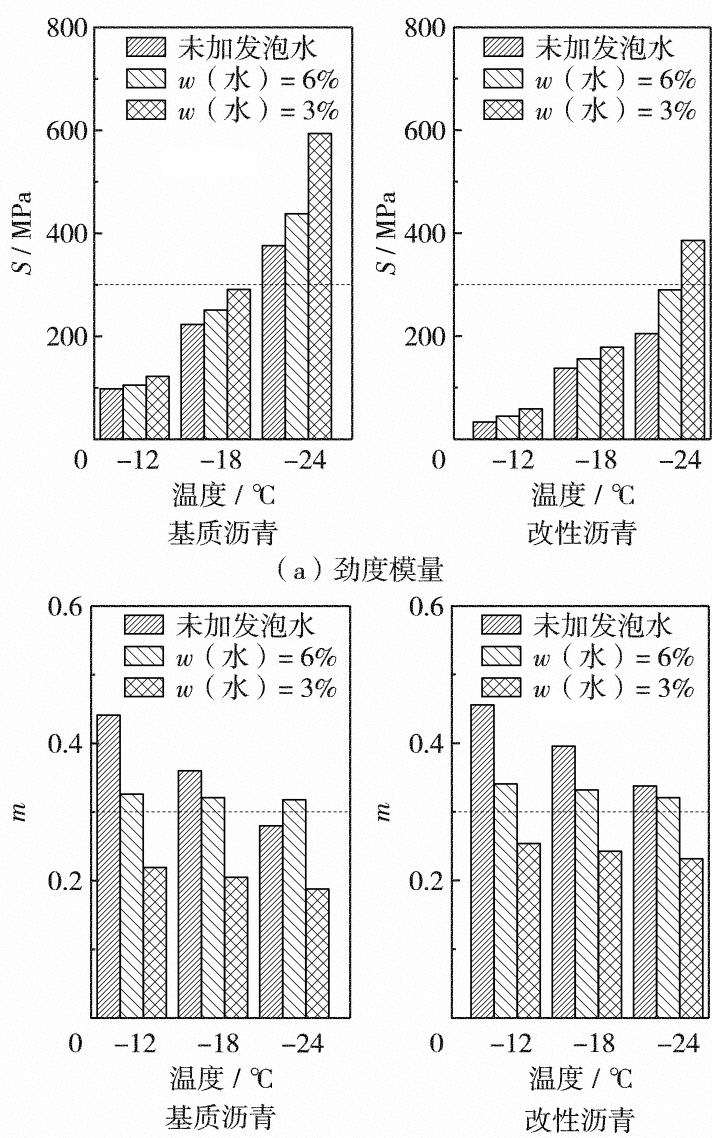

(b ) 蠕变速率

图 3 泡沫温拌沥青的 BBR 试验结果

Fig. 3 BBR test results for foam warm mix asphalt

化的趋势相同. 随着试验温度不断降低，两种沥青 的劲度模量均呈增长趋势, 蠕变速率均呈降低趋 势. 一般而言，沥青的劲度模量越低，沥青低温性 能越好; 蠕变速率越高, 沥青结合料的应力松驰越 好. 这表明随着试验温度降低，两种沥青的低温抗 裂性能有所下降.

当沥青发泡后，从图 3 可见，随着发泡用水量 的不断增大，沥青的劲度模量也在持续增加，而蠕 变速率持续降低. 以 $-24{ }^{\circ} \mathrm{C}$ 为例，当发泡水的质量 分数从 0 分别增至 $3 \%$ 和 $6 \%$ 时，基质沥青的劲度 模量相应增长了 $16.5 \%$ 和 $58.0 \%$ ，蠕变速率相应 降低了 $26.7 \%$ 和 $50.3 \%$; 改性沥青的劲度模量相 应增长了 $41.5 \%$ 和 $85.4 \%$ ，蠕变速率相应降低了 $5.1 \%$ 和 $31.4 \%$. 这反映了发泡水的加人会一定程 度损伤沥青的低温抗裂性能.

与此同时，沥青发泡前后基质沥青和改性沥青 的劲度模量变化率和蠕变速率变化率不一样, 这可 能是由于沥青种类不同，发泡效果也不同引起. 以 $-18{ }^{\circ} \mathrm{C}$ 下的劲度模量为例, $w$ (水) $=3 \%$ 改性沥青 
的劲度模量比未发泡改性沥青高出约 $33.7 \%$, 而同 等发泡水基质沥青发泡后劲度模量增长了约 $7.4 \%$, 说明改性沥青发泡后更容易变脆. 可见，沥青的注 水发泡对低温性能不利，在选取发泡用水量时，应 重点考虑能否满足低温性能要求.

为保证沥青的低温性能，美国 Superpave 规范 要求沥青样品在低温温度下的 $S \leqslant 300 \mathrm{MPa} 、 m>$ $0.3^{[20]}$. 从图 3 可知, 基质沥青符合 $-18^{\circ} \mathrm{C}$ 时的劲 度模量和蠕变速率要求, 但不满足 $-24{ }^{\circ} \mathrm{C}$ 的环境要 求，表明基质沥青的低温符合 $-18{ }^{\circ} \mathrm{C}$ 环境的使用要 求; 加人 $3 \%$ (质量分数) 的水后, 劲度模量有所 增大，蠕变速率有所降低，但仍能符合 $-18{ }^{\circ} \mathrm{C}$ 的低 温要求; 当发泡水继续增至 6\%（质量分数）时, 所有的蠕变速率在 3 种温度下均不能满足，表明 6\% (质量分数) 的发泡水量严重影响了基质沥青 的低温分级, 无法满足 $-12{ }^{\circ} \mathrm{C}$ 的要求; 同样，对于 改性沥青，未发泡及 3\%（质量分数）发泡水的泡 沫沥青, 低温性能符合 $-24{ }^{\circ} \mathrm{C}$ 的环境要求, 泡沫沥 青的低温分级无法满足 $-12{ }^{\circ} \mathrm{C}$ 的环境要求。这表明 了沥青注水发泡削弱了低温抗裂性能，不适用于原 有未发泡沥青的低温适用场合.

为了直观评价沥青材料的低温变化趋势，采用 $m$ 和 $S$ 的比值 $(m / S)$ 来分析. $m / S$ 值越大，表明沥 青材料低温性能越好，结果如图 4. 从图 4 可见， 不同温度、不同沥青类型泡沫温拌沥青的 $m / S$ 值均 小于未发泡沥青，且发泡水量越大，低温性能越 差. 随着温度的降低, $m / S$ 值下降速率逐渐减慢, 这可能是由于温度降低分子, 运动的能量减小, 沥 青中分子的链段运动受到了阻碍，使分子结构趋于 稳定.

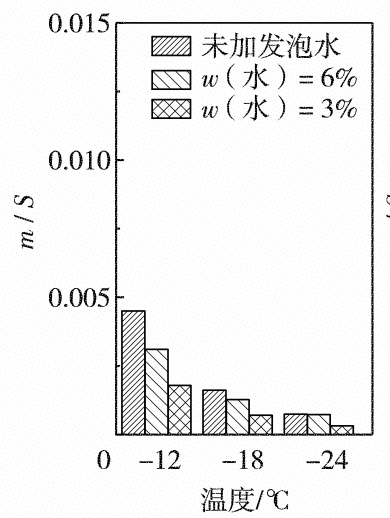

(a) 基质沥青

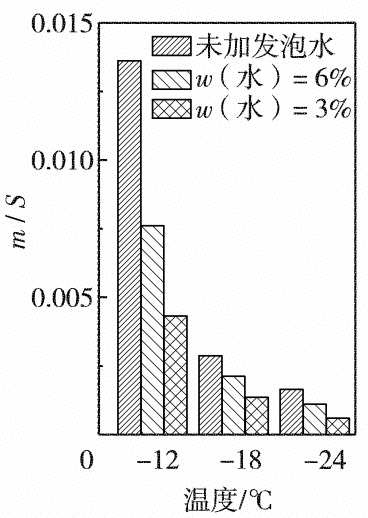

（b）改性沥青
图 4 泡沫温拌沥青的 $m / S$ 结果

Fig. 4 The $m / S$ test results for foam warm mix asphalt

\section{3 结 论}

1) 采用 DMA 和 DSC 方法，对泡沫温拌沥青 进行动态力学性能和热重分析, 得到玻璃态转变温 度. 尽管由两种方法得到的玻璃态转变温度有所区 别，但对于不同的沥青和发泡用水量，两者的变化 趋势一致.

2) 发泡用水量对沥青的玻璃态转变温度有明 显影响,随着发泡水增多,沥青的玻璃态转变温度逐 渐增高, 表明发泡水的加人削弱了沥青的低温性能.

3）随着发泡用水量的不断增大，沥青的劲度 模量持续增大，蠕变速率持续降低，发泡水的加人 会在一定程度上损伤沥青的低温抗裂性能.

4) $3 \%$ (质量分数) 的发泡水尚能与未发泡沥 青保持相同的低温分级，但 6\%（质量分数）的发 泡水下，泡沫温拌沥青不能满足 $-12{ }^{\circ} \mathrm{C}$ 时的低温环 境要求，不适用于原有沥青的应用范围.

$5 ）$ 为保证良好的路用性能,制备泡沫温拌沥青 时, 发泡用水量的选择应重点考虑其低温抗裂能力.

基金项目: 国家自然科学基金资助项目 (51908194); 中国博士后 科学基金资助项目 (2019M650101); 广西重点研发计划 资助项目 (2020AB42005); 江西省重点研发计划资助项 目 (20192BBG70080)

作者简介: 刘圣洁 $(1985-)$, 河海大学副教授、博士. 研究方向 路面工程.E-mail.lsjwork@126.com

引 文: 刘圣洁, 谢政专, 彭爱红. 泡沫温拌沥青的玻璃态转变 温度及低温性能 $[\mathrm{J}]$. 深圳大学学报理工版，2021，38 (2) : $163-169$.

\section{参考文献 / References :}

[ 1 ] LIU Shengjie, ZHOU Shengbo, PENG Aihong, et al. Analysis of the performance and mechanism of desulfurized rubber and low ensity polyethylene compound modified asphalt $[\mathrm{J}]$. Journal of Applied Polymer Science, 2019 6: 48194

[ 2 ] WILliamsB A, COPELAND A, ROSS T C. Asphalt pavement industry survey on recycled materials and warmmix asphalt usage: 2017 [M]. Washington D C: National Asphalt Pavement Association, 2018.

[ 3 ] 林江涛, 梁 皓, 樊 亮. 基于时间及频率扫描的沥青 高低温性能试验研究 $[\mathrm{J}]$. 建筑材料学报, 2018, 21 (3) : 516-522.

LIN Jiangtao, LIANG Hao, FAN Liang. High and low temperature performance test of asphalt based on dynamic shear time and frequency scanning $[\mathrm{J}]$. Journal of Building Materials, 2018, 21 (3): 516-522. (in Chinese) 
[ 4 ] WANG D, FALCHETTO A C, RICCARDI C, et al. Investigation on the low temperature properties of asphalt binder: glass transition temperature and modulus shift factor $[\mathrm{J}]$. Construction and Building Materials, 2020, 245: 118351 .

[ 5 ] 牛 岩, 张晨晨, 王旭东, 等. 基于 DMA 方法的玻璃 态转变温度与沥青混合料低温性能研究 $[\mathrm{J}]$ ．材料导 报, 2017, 31(A02)：484-488.

NIU Yan, ZHANG Chenchen, WANG Xudong, et al. Study on glass transition temperature and low temperature performance of asphalt mixture based on dynamic mechanical analysis method [J]. Materials Review, 2017, 31 (A02) : 484-488. (in Chinese)

[ 6 ] 周维锋. 基于 CAM 复数模量模型的沥青混合料玻璃态 转变温度确定 $[J]$. 公路与汽运, 2019，191(2)：5660,63 .

ZHOU Weifeng. Determination of glass transition temperature of asphalt mixture based on CAM complex modulus model $[\mathrm{J}]$. Highways and Automotive Applications, 2019, 191(2) : 56-60, 63. (in Chinese)

[ 7 ] 颜可珍, 王道珵. 聚合物改性沥青低温性能指标研究 $[\mathrm{J}]$. 建筑材料学报, $2020,120(2)$ ：245-250.

YAN Kezhen, WANG Daocheng. Low temperature performance index of poiymer modified asphalt $[\mathrm{J}]$. Journal of Building Materials, 2020, 120(2) : 245-250. (in Chinese)

[ 8 ] 徐 鹏, 陈华金金, 柇 涛, 等. DSC 试验的彩色沥青相 态转变对比分析 $[\mathrm{J}]$. 材料科学与工程学报, 2019, 37 (4) : 590-594.

XU Peng, CHEN Huaxin, FAN Tao, et al. Analysis of colored asphalt phase transition based on DSC experiment $[\mathrm{J}]$. Journal of Materials Science and Engineering, 2019, 37(4): 590-594. (in Chinese)

[ 9 ] LIU J, SUN Y, WANG W, et al. Using the viscoelastic parameters to estimate the glass transition temperature of asphalt binders $[\mathrm{J}]$. Construction and Building Materials, 2017, 153 : 908-917.

[10] KHANA N, HESP S A M. Comparison between thermal, rheological and failure properties for the performance grading of asphalt cements $[\mathrm{J}]$. Construction and Building Materials, 2019, 220: 196-204.

[11] LIU S, PENG A, ZHOU S, et al. Effect of foaming water on rheological and microscopic properties of foamed warmmix asphalt binders $[\mathrm{J}]$. Journal of Transportation Engineering, Part B: Pavements, 2019, 145(3) : 04019019.

[12] NEWCOMB D E, ARAMBULA E Y. Properties of foamed asphalt for warm mix asphalt applications $[M]$. Washington D C: National Cooperative Highway Research Program, 2015 .
[13] GUO W, GUO X, LI Y, et al. Laboratory investigation on physical, rheological thermal and microscopic characteristics of water-foamed asphalt under three environmental conditions $[\mathrm{J}]$. Coatings, 2020, 10(3) : 239-248.

[14] ALHASAN A A, ABBAS A, NAZZAL M, et al. Lowtemperature characterization of foamed warm-mix asphalt produced by water injection $[\mathrm{J}]$. Civil Engineering Faculty Research, 2014, 2445: 1-11.

[15] 刘 成, 徐 萌, 权 栋, 等. DMA 法研究拌和温度 对沥青黏弹性能的影响 [J]. 中外公路, 2017 (4)： 282-287.

LIU Cheng, XU Meng, QUAN Dong, et al. Study on the influence of DMA mixing temperature on asphalt performance $[\mathrm{J}]$. Journal of China and Foreign Highway, 2017(4) : 282-287. (in Chinese)

[16] 张茂林, 季行行, 史新妍. 聚降冰片烯可逆塑性形状记 忆关键影响因素的研究 $[\mathrm{J}]$. 高分子学报, 2019(9)： 949-956.

ZHANG Maolin, JI Xingxing, SHI Xinyan. Study on the key factors of reversible plasticity shape memory of polynorbornene $[\mathrm{J}]$. Acta Polymerica Sinica, 2019(9) : 949-956. (in Chinese)

[17] 杨 光, 王旭东, 王筵铸, 等. 基于 DMA 方法的沥青 类材料相态转变温度研究 $[\mathrm{J}]$. 公路交通科技, 2019 , $36(3)$ : 33-37.

YANG Guang, WANG Xudong, WANG Yanzhu, et al. Study on phase transition temperature of asphalt materials based on DMA method [ $\mathrm{J}]$. Journal of Highway and Transportation Research and Development, 2019, 36(3): 33-37. (in Chinese)

[18 ] 谭忆秋. 沥青与沥青混合料 $[M]$. 哈尔滨: 哈尔滨工业 大学出版社, 2007.

TAN Yiqiu. Asphalt and asphalt mixture [M]. Harbin: Harbin Institute of Technology Press, 2007. (in Chinese)

[19］吕海宝，杜善义. 聚合物高分子多重玻璃态转变的物 理机制 $[J]$. 现代应用物理，2020(2): 51-60。

LÜ Haibao, DU Shanyi. Multiple glass transitions in polymer macromolecule $[\mathrm{J}]$. Modern Applie Physics, 2020(2) : 51-60. (in Chinese)

[20] 戴文亭, 安 㻆, 郝如意, 等. 疏水性纳米白炭黑对沥 青胶结料低温性能的影响 $[\mathrm{J}]$. 华南理工大学学报自 然科学版, 2020, 48(5): 49-57.

DAI Wenting, AN Yin, HAO Ruyi, et al. Effects of hydrophobic nano-silica on the low-temperature performance of asphalt binder $[\mathrm{J}]$. Journal of South China University of Technology Natural Science, 2020, 48 (5) : 49-57. (in Chinese)

【中文责编：坪 梓; 英文责编：之 聿】 